\title{
Advances in Target Acquisition Modeling
}

\author{
Thomas J. Meitzler \\ Grant R. Gerhart \\ U.S. Army TACOM \\ AMSTA-TR-R, MS 263 \\ Warren, Michigan 48397-5000 \\ Harpreet Singh \\ Wayne State University \\ E\&CE Department \\ 5050 Anthony Wayne Drive \\ Detroit, Michigan 48202
}

\begin{abstract}
The U.S. Army Tank-Automotive Research, Development and Engineering Center (TARDEC) has had a broad interest in modeling and simulation techniques during the last several decades. Specifically, as army ground vehicle designers, our group is interested in predicting the performance of military observers for detecting and discriminating vehicle targets in complex background scenes. We have developed the Visual Perception Laboratory (VPL) to both calibrate and validate target acquisition models for complex visual tasks. This facility is able to augment field test data by presenting visual stimuli to human observers under controlled laboratory conditions. Laboratory perception testing is synergistic with our other modeling and simulation tools in the early test and evaluation phases of vehicle development programs. It provides a means to perform excursions from currently available model and field test data. The model-test-model paradigm is an inherent necessity at the present state of development for target acquisition models.
\end{abstract}

This special section of Optical Engineering contains a selection of papers on the topic Advances in Target Acquisition Modeling. The contributing authors represent a cross section of international experts in this subject area. The majority of the papers deal with some type of military related acquisition task, although much of this work is applicable to commercial applications as well.

The general topic area is quite broad and overlaps with several modeling and simulation research activities including image understanding, expert systems, human psychophysics and object recognition. This collection of papers focuses upon human performance prediction for man-in-the-loop search and target acquisition tasks. It places special emphasis on aided and unaided visual and infrared electro-optical systems. It also complements other special topic sections such as Advances in Recognition Techniques, Parts I and II, which were published in the January and March 1998 issues of Optical Engineering.
Modern military weapon system performance is usually limited by human operator capabilities during battlefield conditions. Operations research models clearly show that the "error bounds" for predicting overall system performance are primarily determined by their ability to accurately predict human performance. Tank commanders or fighter pilots, for example, can often relate their relative success in tactical engagements to the time interval necessary to "acquire the target."

Background clutter and signature countermeasures make these acquisition tasks more difficult by increasing the average target detection and discrimination times. Conversely, aided target recognition or screening devices are designed to improve human performance by reducing these time intervals. In either case human performance capabilities will continue to serve as an important baseline for future semi- or fully-autonomous system requirements and specifications.

Historically the development of robust target acquisition models has been a difficult challenge undertaken primarily by the sensor community. These models have evolved slowly over the years as a consequence of a poor understanding of the human visual system. The methodology has often suffered from two primary deficiencies: (1) a simplistic scene description that attempts to characterize average target/background characteristics but does not adequately address target detail or background clutter, and (2) an almost exclusive emphasis upon empirical extrapolations from psychophysical experiments. The former gives incorrect predictions of sensor performance for realistic background scenes. The latter leads to results that are often tuned to specific visual tasks and can be easily extrapolated beyond their original intended purpose.

The papers in this special section describe models that assume humans employ a variety of visual processes and cognitive strategies. Which mechanisms are operative depends upon the visual task, scene context, and external 
stimuli. Early vision models compute metric values directly from a "front end" retinal-cortical processing module, which takes into account factors such as luminance adaptation, two-stage color vision, multi-scale spatial filtering, temporal filtering, and nonlinear neural receptive field saturation. Some models calculate metrics from simple shape templates, edge curvature weighting, and feature strength vectors using fuzzy logic rules or some other algorithm to predict target acquisition probabilities.

Other papers discuss wide field of regard (WFOR) search and target acquisition (STA) visual tasks for static and dynamic targets. Often times the human observer is viewing a scene through powered optics where the field of view (FOV) of the optical instrument is much different than the original FOR. In this situation there is a tradeoff between optical magnification and a corresponding optimal FOV required to obtain minimum average FOR search times. Optimal STA strategies for unaided observers often involves a combination of foveal and parafoveal vision capabilities. The transition between preattentive and attentive search patterns, for example, is important because the latter leads to longer detection times for difficult to acquire targets.

The more recently developed computational models of early vision are becoming quite comprehensive in scope and include many of the essential elements in the retinocortical neurophysiology. The statistical decision module, however, is inherently empirical at the present time and makes use of a signal decision theory (SDT) paradigm to predict both probabilities of detection and false alarm. The latter is quite important since human detection performance is often limited by false alarm levels for highly cluttered scenes. These false alarm rates are often highly dependent upon observer experience and capabilities, and can be specific to particular background scene content. A very important attribute of SDT in this regard is that the statistical parameter $\mathrm{d}^{\prime}$ compensates for variations in human observer response level for a particular visual task. Other models predict probabilities of detection or hit and ignore false alarms.

A wealth of new information about active brain physiology is coming from techniques such as functional magnetic resonance imaging (fMRI). This knowledge will help reshape our ideas about "smart" image representations that facilitate existing brain machinery to perform complex visual tasks. After the less voluntary early vision system where the elements of the input imagery are processed through structured parallel channels, the "intermediate" vision processes involve image segmentation, shape, and other factors as well such as learning and human experience.

Clearly the more complex visual processes will eventually be understood in terms of bio-mechanisms employed by the brain. This might eventually lead to a knowledge of "involuntary" human brain processes which attempt to make sense out of confusing scenes that contain clutter and other distracters. Many of these issues are discussed among the various papers in this specialsection and will lead to new opportunities for exploiting human target acquisition capabilities by both the sensor and countermeasure designers.
In summary the editors would like to thank the contributing authors for their hard work and fine contributions to the papers in this special section. We would also like to thank Brian Thompson and his staff-especially Karolyn Labes-for their expertise and diligence in helping us with the editorial process. Optical Engineering provides a unique forum in which to organize special topic sections and expeditiously get the technical papers published and distributed to the scientific and engineering community. We have enjoyed the process of working with an exceptional group of international experts in the field of target acquisition modeling and look forward perhaps to doing it once again someday.

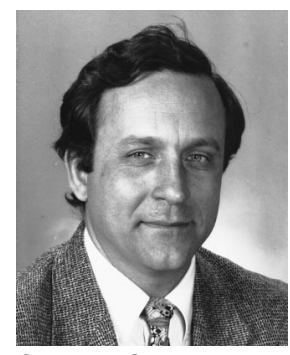

Thomas J. Meitzler received his BS and MS degrees in physics from Eastern Michigan University and a PhD in electrical engineering from Wayne State University in Detroit, Michigan. He has published papers on the application of fuzzy logic and wavelets to human target acquisition modeling and on IR system modeling. He has held teaching positions and the University of MichiganDearborn and Henry Ford Community College. Since 1988 he has been a staff scientist at the U.S. Army TACOM Research and Engineering Center (TARDEC), Survivability Technology Center. His present interests are the validation, verification, and development of electro-optical and visual acquisition models, and experimental visual perception studies in the TARDEC Visual Perception Laboratory. He is a cowinner of the 1995 U.S. Army Research and Development Achievement Award and has coauthored several papers in the fields of IR and visual system simulations.

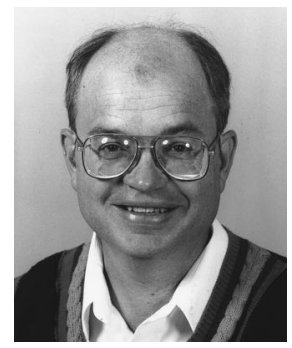

Grant R. Gerhart received a BS degree in physics from lowa State University and a PhD in physics from Wayne State University in Detroit. $\mathrm{He}$ is the author of more than 150 peer-reviewed articles and conference proceedings in acoustics, ultrasonics, holography, image processing, target acquisition and signature modeling, electro-optical sensor effects, and ground vehicle robotics technology. $\mathrm{He}$ also holds five patents, is program manager for the TARDEC in-house laboratory research program (ILIR), and participates on several NATO committees and international bi-lateral expert groups in ground vehicle survivability technology. He is a co-chair of the SPIE AeroSense technical conference on Robotic and Semi-robotic Ground Vehicle Technology, and is currently involved in the analysis of polarized light for signature analysis applications and early vision models for target detection and discrimination.

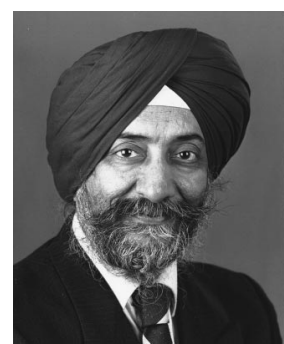

Harpreet Singh received his BSc in engineering from Punjabi University in 1963 and his PhD in electrical engineering from the University of Roorkee, India, in 1971. He was with the Electronics and Engineering Department of the University of Roorkee from 1963 to 1981, where he developed a postgraduate program in computer engineering and received the Khosla Award (highest) in 1971. He joined Wayne State University in 1981, where he is presently a professor. He has published over 200 papers in international journals and publications and has been the associate chair of the Department of Electrical and Computer Engineering for several years. His current interests are computer vision and target detection, system theory, fuzzy and neural networks, and software engineering. 